\title{
Changes in disease gene frequency over time with differential genotypic fitness and various control strategies
}

\author{
P. N. Thompson, ${ }^{1}$ J. A. P. Heesterbeek, ${ }^{2}$ and J. A. M. van Arendonk ${ }^{3}$ \\ Department of Production Animal Studies, Faculty of Veterinary Science, University of Pretoria, \\ Onderstepoort, 0110, South Africa
}

\begin{abstract}
A spreadsheet model was constructed to describe the change in allelic frequency over time for a lethal recessive mutation in an animal population. The model allowed relative fitness to differ between genotypes, between sexes, and over time. Whereas a lethal recessive allele is naturally eliminated very slowly from a population, a small selective disadvantage of the heterozygote results in a large increase in the rate of elimination. With selective advantage of the heterozygote through linkage with a production trait or pleiotropy, the allele is never naturally eliminated but tends toward a stable equilibrium frequency. The model was used to investigate various alternative con-
\end{abstract}

trol programs based on the detection of heterozygotes by genotyping and their exclusion from breeding. The programs (genotyping males only, genotyping males and $50 \%$ of females, and genotyping all breeding animals) were modeled for various initial heterozygote frequencies, and the results were described in terms of the number of generations, number of tests, and number of culls required to reduce the heterozygote frequency to a predefined level. The model can be used to compare the feasibility and cost of various control strategies and to illustrate clearly to breeders the expected outcomes, as well as the danger of prematurely terminating a control program when there is a selective advantage of the heterozygote.

Key words: fitness, genetic defect, genotyping, lethal recessive mutation, modeling, selective advantage of heterozygote

(2006 American Society of Animal Science. All rights reserved.

J. Anim. Sci. 2006. 84:2629-2635

doi:10.2527/jas.2005-774

\section{INTRODUCTION}

Several hundred single-locus defects have been described in domestic animals, many of which are lethal autosomal recessives (Nicholas, 2005). These conditions may be economically important, and there are several accounts of attempts to eradicate such mutations (Jolly, 1975; Robinson et al., 1993; Hogasen et al., 1997). Control programs depend largely on the ability to detect heterozygotes, complete removal of which from the breeding population will ensure eradication of the mutant allele. For some mutations, the availability of specific PCR tests (Bilstrom et al., 1998; Dennis et al., 2000; Kraner et al., 2002) enables rapid and accurate detection of carriers. However, in practice it is often not possible to genotype all breeding animals.

\footnotetext{
${ }^{1}$ Corresponding author: peter.thompson@up.ac.za

${ }^{2}$ Department of Farm Animal Health, Faculty of Veterinary Medicine, Utrecht University, the Netherlands.

${ }^{3}$ Animal Breeding and Genetics Group, Wageningen University, the Netherlands.

Received December 30, 2005.

Accepted May 16, 2006.
}

In most autosomal recessive conditions, heterozygotes are phenotypically indistinguishable from normal homozygotes, and relative fitness of the 2 genotypes is assumed to be equal. However, if there is a difference in phenotype of the heterozygote, due to an effect of the mutant allele itself (pleiotropy) or to close linkage with another gene affecting form or function, selection for or against the heterozygote may occur, altering its relative fitness and therefore its change in frequency from one generation to the next.

When considering alternatives for the eradication or control of a genetic disease, it is important to be able to predict changes in gene frequency over time. This can be calculated using formulas to account for differences in relative fitness between genotypes and between sexes (Hartl and Clark, 1997; Hedrick, 2000). Alternatively, and more flexibly, it can be iteratively modeled from one generation to the next, allowing fitness also to vary over time and providing graphical depiction of changes.

The objective of this paper was to describe the changes in frequency of a lethal autosomal recessive mutation over time in a spreadsheet model and to use it to predict the results of various control strategies. 


\section{MATERIALS AND METHODS}

A single biallelic locus, with alleles $A$ and $a$, was considered, with $A$ representing the wild-type allele and $a$ representing the allele carrying the mutation. The frequency of allele $A$ was denoted by $p$ and that of allele $a$ by $q=1-p$; the initial ratio of $A A: A a: a a$ in the base population at birth was therefore $p^{2}: 2 p q: q^{2}$, assuming Hardy-Weinberg equilibrium with no selection, new mutation, or migration. Henceforth, and for all subsequent generations, individuals underwent natural or artificial selection or both and survived to breeding with probabilities defined by the relative fitness of their particular genotype. The relative fitnesses of the $A A, A a$, and $a a$ genotypes were denoted by $w_{A A}, w_{A a}$, and $w_{a a}$, respectively $\left(\boldsymbol{w}_{\boldsymbol{A A m}}, \boldsymbol{w}_{\text {Aam }}\right.$, and $\boldsymbol{w}_{\text {aam }}$ for males, and $\boldsymbol{w}_{\boldsymbol{A} \boldsymbol{A} \boldsymbol{f}}, \boldsymbol{w}_{\boldsymbol{A} \boldsymbol{a} \boldsymbol{f}}$, and $\boldsymbol{w}_{\boldsymbol{a a f}}$ for females). A selective advantage of the heterozygote over the wild-type homozygote existed if $w_{A a}>w_{A A}$, and a selective disadvantage of the heterozygote existed if $w_{A a}<w_{A A}$.

The frequency of each genotype at birth was thus multiplied by its relative fitness, producing a ratio $A A: A a: a a$ among adult males of $p^{2} w_{A A m}: 2 p q w_{A a m}: q^{2-}$ $w_{\text {aam }}$ and among adult females of $p^{2} w_{A A f}: 2 p q w_{A a f}: q^{2} w_{a a f}$. Each value (i.e., product of genotypic frequency at birth and relative fitness) was standardized by dividing it by the sum of the values for the 3 genotypes (also known as the mean fitness value) to obtain breeding adult genotypic frequencies, e.g., the frequency of the $A a$ genotype among adult males after selection was $2 p q w_{\text {Aam }} /$ $\left(p^{2} w_{A A m}+2 p q w_{\text {Aam }}+q^{2} w_{\text {aam }}\right)$. After selection, mating was assumed to be random. Offspring genotypic and allelic frequencies were then calculated for each subsequent generation, assuming Mendelian segregation.

The model further assumed infinite population size, nonoverlapping generations, constant fitness over time for any specified genotype, and that no further mutations occurred in the same gene. For the control strategies, it was assumed that an accurate test existed to distinguish between heterozygotes and wild-type normal homozygotes.

The model was constructed using a commercial spreadsheet program (Excel 2003, Microsoft Corporation, Redmond, WA). Inputs were initial allelic frequencies, relative fitnesses for each genotype/sex combination, numbers of males and females selected for breeding, and for the control strategies, proportions of males and females genotyped. Outputs for each subsequent generation were genotypic and allelic frequencies at birth and after selection for breeding, and for the control strategies, the expected number of tests and culls required to obtain the desired number of noncarrier breeding males and females, as well as cumulative totals.

\section{Base Scenarios}

Models were generated for different scenarios by varying the initial mutant allelic frequency $q(0.4,0.1$, and
0.01 ) and the relative fitness $w$ for each genotype in males and females. The scenarios and fitness ratios $\left(w_{A A}: w_{A a}: w_{a a}\right)$ considered were lethal recessive, 1:1:0 (scenario 1); lethal recessive with selective advantage of the heterozygote, 1:1.1:0 (scenario 2) and 1:1.5:0 (scenario 3); and lethal recessive with selective disadvantage of the heterozygote, 1:0.9:0 (scenario 4) and 1:0.5:0 (scenario 5). The results of each model were expressed in terms of the time required to reduce $q$ by 50 and $90 \%$, and the equilibrium frequency reached, if any.

\section{Control Strategies}

The model was then used to investigate strategies for the eradication of a lethal autosomal recessive mutation in a hypothetical animal population of 15,000 breeding females and 1,200 breeding males. It was assumed that an accurate genotype test was available and that every heterozygous animal tested was excluded (culled) from the breeding population. Thus, in each generation the required number of candidate males or females or both were tested to select the next breeding generation of 15,000 females and 1,200 males. The model was run using various starting values $(0.4,0.1$, and 0.01 ) for the prevalence of heterozygotes in the base generation.

The control strategies were modeled by varying the relative fitness of each genotype in males and females. For example, a genotype that was culled was assigned a fitness of zero. The options considered for the eradication of the mutation were as follows:

Genotyping of males only: All breeding males were certified as noncarriers, but females were not tested, $w_{A A m}: w_{\text {Aam }}: w_{\text {aam }}=1: 0: 0$ and $w_{A A f}: w_{\text {Aaf }}: w_{\text {aaf }}=1: 1: 0$ (strategy F).

Genotyping of males and partial genotyping of females: All breeding males but only half of the breeding females were certified as noncarriers, $w_{A A m}: w_{\text {Aam }}: w_{\text {aam }}=1: 0: 0$ and $w_{A A f}: w_{A a f}: w_{a a f}=1: 0.5: 0$ (strategy G).

Genotyping of all breeding animals: Breeding males and females were all certified to be noncarriers, $w_{A A m}: w_{A a m}: w_{\text {aam }}=w_{A A f}: w_{A a f}: w_{\text {aaf }}=1: 0: 0$ (strategy H).

In addition, the scenario was considered of a lethal recessive with selective advantage of the heterozygote, $w_{A A}: w_{A a}: w_{a a}=1: 1.5: 0$, in which genotyping of breeding males only was performed (strategy I). In this case, the effect of terminating the control program after 5 generations was also investigated (strategy J).

Two alternative endpoints for each strategy were used:

Attainment of an arbitrary low prevalence $(\leq 0.1 \%)$ of heterozygotes in the population.

Eradication, defined as being $95 \%$ certain that no carriers will be present among the 16,200 animals selected 
Table 1. Changes in allelic frequency over time with differing genotypic fitnesses and initial allelic frequencies

\begin{tabular}{|c|c|c|c|c|c|}
\hline \multirow[b]{2}{*}{ Type } & \multirow{2}{*}{$\begin{array}{c}\text { Relative } \\
\text { fitnesses of } \\
A A: A a: a a\end{array}$} & \multirow{2}{*}{$\begin{array}{c}\text { Initial } \\
\text { frequency } q \\
\text { of allele } a\end{array}$} & \multicolumn{2}{|c|}{$\begin{array}{l}\text { Generations required to } \\
\text { reduce frequency by: }\end{array}$} & \multirow{2}{*}{$\begin{array}{l}\text { Equilibrium } \\
\text { frequency } \hat{q} \\
\text { of allele } a\end{array}$} \\
\hline & & & $50 \%$ & $90 \%$ & \\
\hline \multirow[t]{2}{*}{ Lethal recessive } & $1: 1: 0$ & 0.1 & 10 & 90 & 0 \\
\hline & (Scenario A) & 0.01 & 100 & 900 & 0 \\
\hline \multirow{6}{*}{$\begin{array}{l}\text { Lethal recessive with selective } \\
\text { advantage of heterozygote }\end{array}$} & 1:1.1:0 & 0.4 & 4 & $-^{1}$ & 0.083 \\
\hline & (Scenario B) & 0.1 & - & - & 0.083 \\
\hline & & 0.01 & - & - & 0.083 \\
\hline & $1: 1.5: 0)$ & 0.4 & - & - & 0.25 \\
\hline & (Scenario C) & 0.1 & - & - & 0.25 \\
\hline & & 0.01 & - & - & 0.25 \\
\hline \multirow{4}{*}{$\begin{array}{l}\text { Lethal recessive with selective } \\
\text { disadvantage of heterozygote }\end{array}$} & 1:0.9:0 & 0.1 & 5 & 18 & 0 \\
\hline & (Scenario D) & 0.01 & 7 & 22 & 0 \\
\hline & $1: 0.5: 0$ & 0.1 & 1 & 4 & 0 \\
\hline & (Scenario E) & 0.01 & 1 & 4 & 0 \\
\hline
\end{tabular}

${ }^{1}$ Not possible because equilibrium frequency was reached.

for breeding. This would be the case when the prevalence of carriers among animals available for selection is less than $0.0003 \%$. This value was obtained using the binomial distribution

$$
\begin{gathered}
P(0,16,200,0.000003)= \\
\left(\begin{array}{c}
16,200 \\
0
\end{array}\right) \times 0.000003^{0} \times(1-0.000003)^{16,200-0} \approx 0.95
\end{gathered}
$$

For each control strategy, the number of generations, cumulative number of tests, and cumulative number of culls required to achieve the above endpoints were calculated.

\section{RESULTS}

The number of generations required to reduce the frequency of the mutant allele by 50 and $90 \%$, and the equilibrium frequency reached, if any, are shown in Table 1. The changes in allelic frequency over time are shown in Figure 1. When the heterozygote has no selective disadvantage compared with the wild-type homozygote (A), a lethal recessive allele is eliminated very slowly from a population. With selective advantage of the heterozygote (B and C), the frequency of $a$ reaches a stable equilibrium, which is independent of the initial allelic frequency but dependent on the relative fitness of the 3 genotypes. In such cases, the recessive allele will never be eliminated naturally from a population, but will persist at an expected equilibrium frequency $\hat{q}=\left(w_{A a}-w_{A A}\right) /\left(2 w_{A a}-w_{a a}-w_{A A}\right)$ (Hartl and Clark, 1997). In contrast, a small selective disadvantage of the heterozygote (e.g., D: $w_{A a}=0.9$ ) results in a large increase in the rate at which the allelic frequency is reduced. If the relative fitness of the heterozygote is further reduced (e.g., E: $w_{A a}=0.5$ ), the allelic frequency declines very rapidly, irrespective of the initial frequency.
Tables 2 and 3 show the number of generations that would be required to reduce the prevalence of heterozygotes in our hypothetical population to below 0.1 and $0.0003 \%$, respectively, using various test and cull strategies and for different initial heterozygote prevalences. For each strategy, the expected cumulative numbers of animals required to be genotyped and culled over the duration of the program are also shown. For example, at an initial carrier prevalence of $10 \%$, testing all males and culling all carriers (strategy $\mathrm{F}$ ) will reduce the carrier prevalence to $<0.1 \%$ over 7 generations, requiring 8,654 tests and the culling of 254 carrier males. Testing and culling females rather than males will achieve the same rate of reduction, but with a more than 10 -fold increase in the number of tests and culls required (results not shown). Testing all males with partial (50\%) implementation of testing of breeding females (strategy

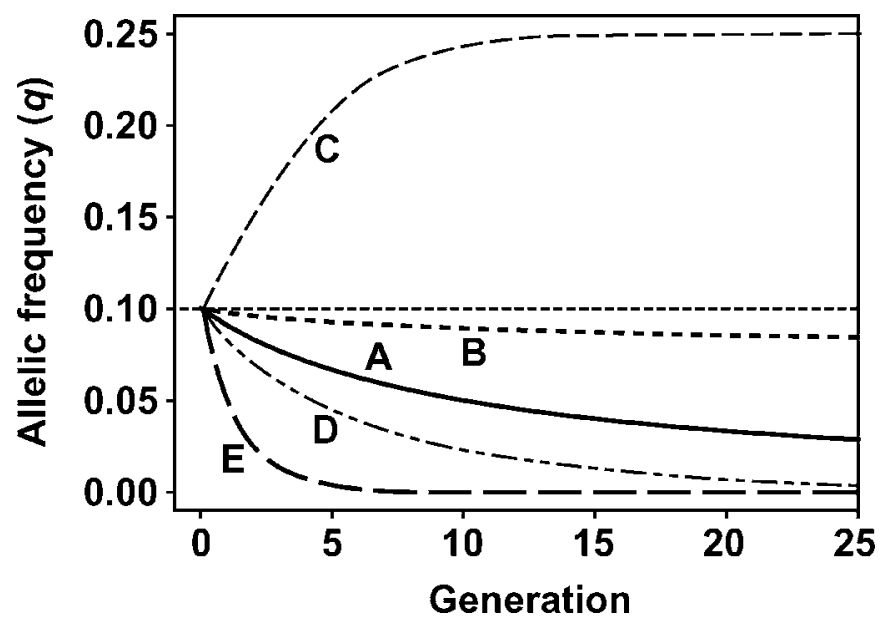

Figure 1. Changes in recessive allelic frequency over 25 generations with different relative genotypic fitnesses. Relative genotypic fitnesses $\left(w_{A A}: w_{A a}: w_{a a}\right)$ are as follows: $\mathrm{A}=1: 1: 0 ; \mathrm{B}=1: 1.1: 0 ; \mathrm{C}=1: 1.5: 0 ; \mathrm{D}=1: 0.9: 0 ; \mathrm{E}=1: 0.5: 0$. 
Table 2. Characteristics of alternative eradication strategies to reduce carrier prevalence to below $0.1 \%$ for a lethal recessive mutation in a population of 15,000 females and 1,200 males, for different relative genotypic fitnesses $\left(w_{A A}: w_{A a}: w_{a a}\right)$ and initial carrier prevalences $(2 p q)$

\begin{tabular}{|c|c|c|c|c|c|c|c|c|c|}
\hline \multirow{2}{*}{$\begin{array}{l}\text { Relative } \\
\text { fitness } \\
\text { AA:Aa:aa }\end{array}$} & \multirow[b]{2}{*}{ Strategy } & \multirow{2}{*}{$\begin{array}{c}\text { Initial } \\
\text { carrier } \\
\text { prevalence, } \\
\%\end{array}$} & \multicolumn{7}{|c|}{ Required to reduce carrier prevalence to below $0.1 \%$} \\
\hline & & & Generations & Male & Female & Total & Male & Female & Total \\
\hline \multirow[t]{7}{*}{ 1:1:0 } & $\mathrm{F}:$ & 40 & 9 & 12,114 & 0 & 12,114 & 1,314 & 0 & 1,314 \\
\hline & Test and cull males & 10 & 7 & 8,654 & 0 & 8,654 & 254 & 0 & 254 \\
\hline & & 1 & 4 & 4,823 & 0 & 4,823 & 23 & 0 & 23 \\
\hline & & 1 & 2 & 2,416 & 15,095 & 17,511 & 16 & 95 & 111 \\
\hline & $\mathrm{H}:$ & 40 & 1 & 2,000 & 25,000 & 27,000 & 800 & 10,000 & 10,800 \\
\hline & Test and cull all males and females & 10 & 1 & 1,334 & 16,667 & 18,000 & 134 & 1,667 & 1,800 \\
\hline & & 1 & 1 & 1,213 & 15,152 & 16,365 & 13 & 152 & 165 \\
\hline \multirow[t]{2}{*}{ 1:1.5:0 } & I: & 40 & 19 & 24,681 & 0 & 24,681 & 1,881 & 0 & 1,881 \\
\hline & Test and cull males & 10 & 16 & 19,660 & 0 & 19,660 & 460 & 0 & 460 \\
\hline
\end{tabular}

G) will greatly reduce the time required to achieve the same objective, resulting in fewer males (176) being culled, but requires the testing and culling of a large number of females. If the carrier has a selective advantage, eradication is a far longer and more costly process (strategy I). However, if all breeding animals are tested, the selective advantage is of no consequence, and it becomes equivalent to strategy $\mathrm{H}$, in which the mutant allele is eliminated from the population within 1 generation.

Figure 2 illustrates the expected changes in heterozygote prevalence over the first 10 generations for each strategy, compared with no control program (N), starting at a carrier frequency of $10 \%$, with no heterozygote advantage (N, F, G, and $\mathrm{H}$ ) and with selective advantage of the heterozygote (I). The mutant allele is completely eradicated from the population only in eradication strategy $\mathrm{H}$ (i.e., ensuring that no carriers are used for breeding). In all other strategies the carrier frequency is merely reduced. To illustrate the latter point, the effect of discontinuing a control program when a heterozygote selective advantage exists is shown in Figure 2 (line J). In such a situation, the frequency of the mutant allele begins to increase again toward the equilibrium frequency $\hat{q}$.

\section{DISCUSSION}

Our results show that the outcome of a program to eradicate a lethal recessive from a population depends greatly on the fitness value of the heterozygote (Tables 2 and 3). This is in line with results in the literature (Lefort and Lauvergne, 1974; Hoeschele and Meinert, 1990).

Table 3. Characteristics of alternative eradication strategies to reduce carrier prevalence to below $0.0003 \%$ for a lethal recessive mutation in a population of 15,000 females and 1,200 males, for different relative genotypic fitnesses $\left(w_{A A}: w_{A a}: w_{a a}\right)$ and initial carrier prevalences $(2 p q)$

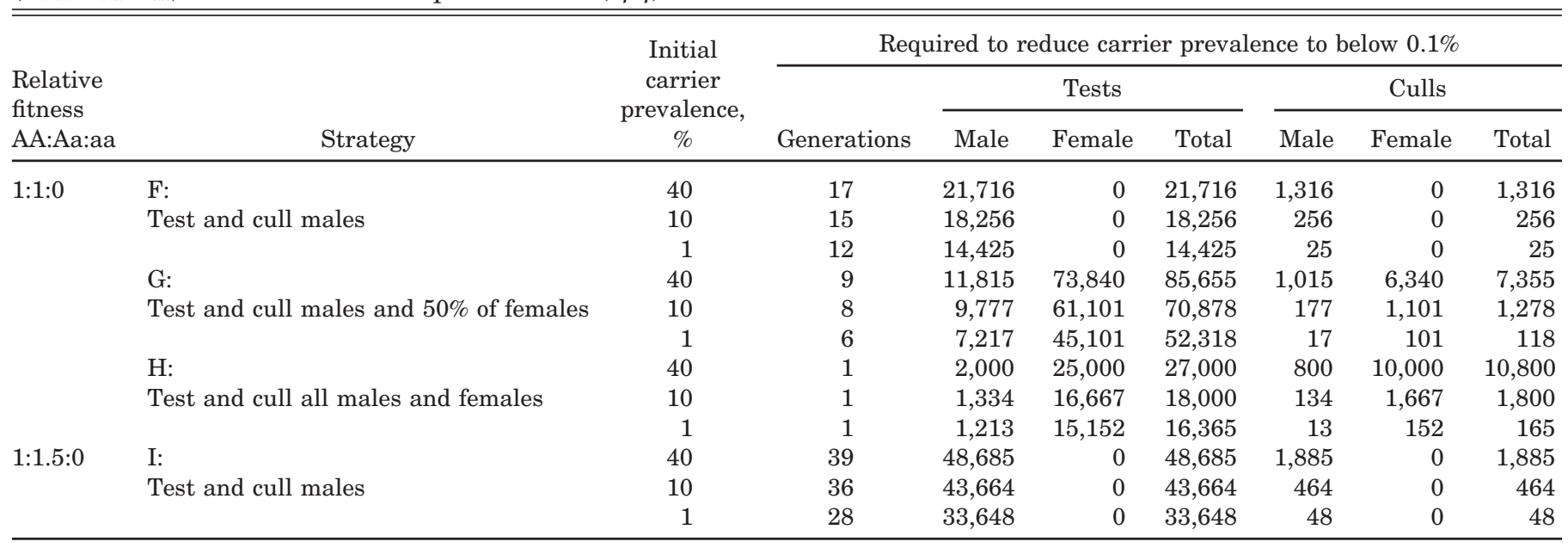




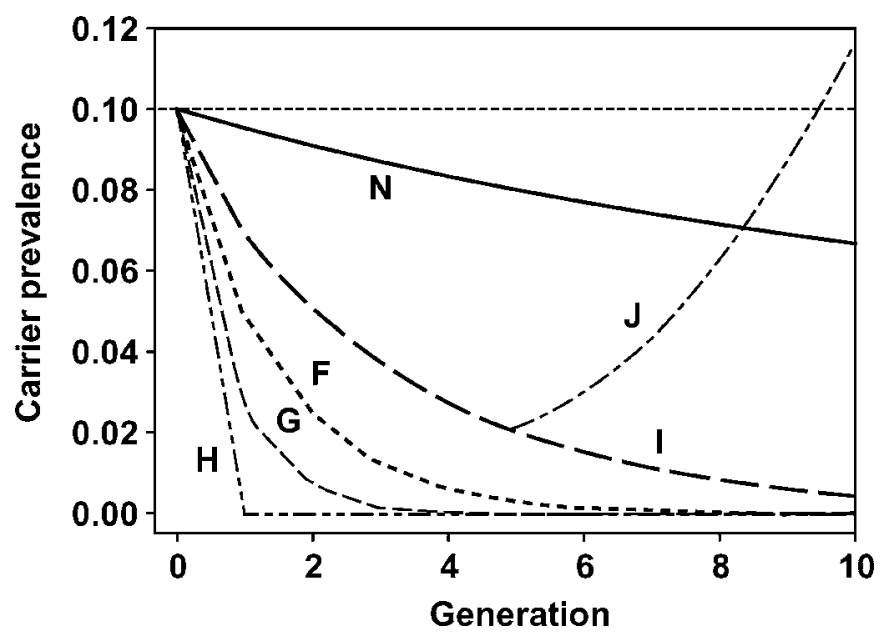

Figure 2. Changes in prevalence of carriers over 10 generations using various strategies for the eradication of a lethal recessive allele. $\mathrm{N}=$ no control program; $\mathrm{F}=$ testing males only and culling carriers; $\mathrm{G}=$ testing males and $50 \%$ of females and culling carriers; $\mathrm{H}=$ testing all breeding animals and culling carriers; $\mathrm{I}=$ testing of males only and culling carriers (selective advantage of heterozygote); J = as for I, but testing and culling ceases after 5 generations. Relative genotypic fitnesses $\left(w_{A A}: w_{A a}: w_{a a}\right)$ are 1:1:0 for N, F, G, and H; and 1:1.5:0 for I and J.

\section{Differential Genotypic Fitness}

A selective advantage or disadvantage of the heterozygote over the wild-type homozygote can also be quantitatively expressed as $w_{A a}-w_{A A}=s$, the selection coefficient (Hedrick, 2000). In this model, however, only the relative fitness values of the 3 genotypes were specified. This implies the assumption that the average fitness value was sufficiently large to maintain a stable population size.

Examples of lethal recessive conditions in cattle where there is no apparent heterozygote advantage include $\alpha$-mannosidosis in Angus and Murray Greys in Australia and New Zealand (Jolly et al., 1974), generalized glycogenosis in Brahmans (Dennis et al., 2000), and myophosphorylase deficiency in Charolais (Bilstrom et al., 1998). In humans, the majority of inherited defects, and particularly autosomal recessive defects, are expressed early in life, before puberty (Costa et al., 1985), although there are several autosomal recessive disorders, e.g., some forms of parkinsonism (Lincoln et al., 2003), with a much later age of onset. The same is likely also to be true in animals, where a mutation causing a visible disease or defect, even if not lethal, will usually result in the animal not being selected for breeding. Thus, the fitness of the disease genotype will effectively be zero.

In some recessive disease conditions, the heterozygote has a selective advantage over the homozygous wild-type animal due to a pleiotropic effect or close linkage with genes affecting a trait under selection.
Selection on that trait will therefore increase the fitness of the heterozygote relative to that of the homozygous wild-type animal. For example, Brown Swiss cows carrying the weaver gene have a greater milk yield (Hoeschele and Meinert, 1990), Brahman cattle carrying the CHRNE 470del20 mutation causing congenital myasthenic syndrome have increased $600 \mathrm{~d}$ weight (P. N. Thompson, unpublished data), and carriers of the halothane gene causing malignant hyperthermia in pigs show better feed efficiency, and greater carcass yield and carcass lean content (Leach et al., 1996). Selective advantage of the heterozygote may also be due to a pleiotropic effect of the recessive allele itself. In humans, carriers of sickle-cell anemia (Aidoo et al., 2002) and cystic fibrosis (Gabriel et al., 1994; Schroeder et al., 1995) may have a selective advantage due to increased resistance to other diseases.

Selective disadvantage of the heterozygote may also occur. In chondrodysplasia of Dexter cattle, the heterozygote is easily recognized by shortness of the limbs, whereas the recessive homozygote shows lethal chondrodysplasia (Harper et al., 1998). In these and other conditions in which the heterozygote is phenotypically different from the wild-type homozygote, the relative fitness of the heterozygote will depend on breeders' preferences for phenotypes and their awareness of the genetic defect. Selective disadvantage of the heterozygote may also occur because of close linkage with genes affecting production traits or pleiotropy. An example of the latter is the finding that fresh meat quality is poorer in carriers of the halothane gene in pigs (Moelich et al., 2003). Carriers of the mutation responsible for bovine factor XI deficiency show a partial factor XI deficiency, which may negatively affect survival or productivity (Brush et al., 1987). In chondrodysplasia of Hereford and Angus cattle, heterozygotes show skeletal abnormalities but to a much lesser degree than those seen in affected calves (Emmerson and Hazel, 1956). In the case of bovine leukocyte adhesion deficiency, a significant negative association of the mutant allele with milk protein yield has been reported (Powell et al., 1996); however, it is not clear whether this is due to pleiotropy or close linkage. Another mechanism by which heterozygotes may be at a selective disadvantage is if the homozygous recessive genotype results in embryonic or fetal loss, resulting in the breeder selecting against the heterozygous dam. This occurs in inherited deficiency of uridine monophosphate synthase in Holstein cattle, where homozygous recessive embryos are lost at about d 40 of gestation (Shanks and Robinson, 1989). In general, recessive diseases with selective disadvantage of the heterozygote are likely to be less common than those with selective advantage of the heterozygote because, as shown in the model, such mutations will decline in frequency relatively rapidly. A small decrease in performance will also be amplified by selection on that trait, resulting in a bigger decrease in relative fitness and a more rapid decline in frequency of the recessive allele. This also means that it is difficult for such a mutation to 
reach a high frequency in the population after initially occurring in a single animal. An exception would be if the mutation was present in an influential sire, in which case the allelic frequency would initially reach 0.25 among its progeny.

\section{Elimination of Recessive Alleles}

It is well known that a recessive lethal gene, with no heterozygote advantage or disadvantage, will naturally be eliminated very slowly from a population because most copies of the recessive allele occur in heterozygotes. The frequency $\left(q_{t}\right)$ of allele $a$ after $t$ generations can be calculated as $q_{t}=q_{0} /\left(1-t q_{0}\right)$ (Hedrick, 2000); by rearrangement the time required to reduce the allelic frequency from $q_{0}$ to $q_{t}$ can be calculated as $t=\left(1 / q_{t}\right)-$ $\left(1 / q_{0}\right)$. However, this is valid only when there is no heterozygote advantage. Various other equations can be used to calculate $q_{t}$, depending on whether the mutant allele is recessive, additive, or dominant (Hedrick, 2000). Our simulation model enabled studying the dynamics of the mutant allele using any desired combination of relative fitnesses, including differences in relative fitness between sexes and variation in relative fitnesses over time.

To prevent the occurrence of homozygote recessives, it is sufficient to test all parents from one sex to ensure that no carriers are used for breeding. The model shows that the effect of genotyping all females only is the same as that of genotyping all males only, except that it requires more testing and culling. It therefore makes economic sense to test at least all parents of the sex with the smallest number of individuals (i.e., sires in most cases). However, if one wants to be sure of eradicating the mutant allele, rather than just preventing the occurrence of disease, then it is clear that both sexes need to be genotyped (Tables 2 and 3). This is particularly important when carriers have a selective advantage over homozygous wild type animals, because eradication is slower and a break in the testing program may result in the frequency of the mutant allele increasing again. Partial testing of females, in addition to testing of males, increases the rate of elimination of the mutation. Although not considered in this model, it may be more cost-effective to target this testing to include only those females used in the production of future breeding males.

With a selective advantage of the heterozygote, the eventual equilibrium frequency $\hat{q}$ of the mutant allele can be predicted using $\hat{q}=\left(w_{A a}-w_{A A}\right) /\left(2 w_{A a}-w_{a a}-\right.$ $w_{A A}$ ) (Hartl and Clark, 1997). As expected, our simulation model yields the same results for $\hat{q}$, with the advantage that it permits the relative fitnesses to differ between sexes. This may be the case in sex-limited traits and when there is a difference in the way the 2 sexes are tested and culled, as in strategies F, G, and I.

Another advantage of the simulation model described here is that estimates can be made of the costs of implementing a control program on a generation by genera- tion basis, and alternative control strategies can easily be evaluated. An example of a cost-benefit analysis was demonstrated by Jolly and Townsley (1980) for the bovine mannosidosis control program. The cost of obtaining genotype information can be substantially reduced by using segregation analysis to calculate genotype probabilities and genotype probability indices for untested individuals based on known genotypes of relatives (Kerr and Kinghorn, 1996). This, together with pedigree information, can then be used to select for further testing those individuals with the poorest genotype information, and which would contribute the most genotype information to the rest of the population (Kinghorn, 1999).

In our model we used nonoverlapping generations, but it also provides good insight for situations with overlapping generations. Eradication programs focus on identifying and eliminating carriers before animals have reached their reproductive age. The number of years for which individual animals are used for breeding (i.e., whether generations overlap) will not affect the change in gene frequency over generations (Moran, 1958; Jansen et al., 1984), but it will affect the translation from generations into time (i.e., years). It is possible to extend the model to account for overlapping generations, but this will not substantially change the conclusions.

An important part of the planning of a control program is clear communication and presentation of the anticipated outcomes to the breeders, breed society, or other interested parties. Using a simulation model, projected changes in carrier and allelic frequencies over time can easily be demonstrated and compared between alternative control programs. The potential danger of premature termination of a program can also be demonstrated.

It is important to monitor the level of inbreeding when eliminating carriers of a mutant allele, particularly when the initial carrier frequency is high, when the population is small, and when the wild-type allele comes from a limited number of ancestral families (Sonesson et al., 2003). Elimination of carriers may lead to an undesired increase in inbreeding, which, in turn, may lead to an undesired increase in the frequency of other genetic defects. An alternative to culling a carrier male would be to use it to produce a group of offspring and then to genotype and cull those that are carrying the mutant allele. Although more expensive, this would reduce the loss of genetic diversity.

\section{IMPLICATIONS}

Recessive mutations causing disease are naturally eliminated very slowly from animal populations because most copies of the mutant allele are hidden in apparently normal carriers. However, sometimes their performance may differ from that of noncarriers, resulting in a selective disadvantage, with far more rapid elimination of the mutation, or a selective advantage, 
where the mutation is never eliminated but reaches a stable equilibrium frequency. Our model can be used to predict the cost and time required to eradicate a recessive mutation under these conditions, using various strategies. To prevent disease due to homozygote recessives, it is sufficient to test all members of one sex (usually males) and avoid breeding with carriers. But to be sure of eliminating the mutation from the population, it is necessary to test all breeding animals. If carriers have a selective advantage, termination of testing is expected to result in an increase in the frequency of the mutant allele.

\section{LITERATURE CITED}

Aidoo, M., D. J. Terlouw, M. S. Kolczak, P. D. McElroy, F. O. ter Kuile, S. Kariuki, B. L. Nahlen, A. A. Lal, and V. Udhayakumar. 2002. Protective effects of the sickle cell gene against malaria morbidity and mortality. Lancet 359:1311-1312.

Bilstrom, J. A., S. J. Valberg, D. Bernoco, and J. R. Mickelson. 1998. Genetic test for myophosphorylase deficiency in Charolais cattle. Am. J. Vet. Res. 59:267-270.

Brush, P. J., P. H. Anderson, and R. F. Gunning. 1987. Identification of factor XI deficiency in Holstein-Friesian cattle in Britain. Vet. Rec. 121:14-17.

Costa, T., C. R. Scriver, and B. Childs. 1985. The effect of Mendelian disease on human health: A measurement. Am. J. Med. Genet. 21:231-242

Dennis, J. A., C. Moran, and P. J. Healy. 2000. The bovine $\alpha$-glucosidase gene: Coding region, genomic structure, and mutations that cause bovine generalized glycogenosis. Mamm. Genome 11:206-212.

Emmerson, M. A., and L. N. Hazel. 1956. Radiographic demonstration of dwarf gene-carrier beef animals. J. Am. Vet. Med. Assoc. 128:381-390.

Gabriel, S. E., K. N. Brigman, B. H. Koller, R. C. Boucher, and M. J. Stutts. 1994. Cystic fibrosis heterozygote resistance to cholera toxin in the cystic fibrosis mouse model. Science 266:107-109.

Harper, P. A., M. R. Latter, F. W. Nicholas, R. W. Cook, and P. A. Gill. 1998. Chondrodysplasia in Australian Dexter cattle. Aust. Vet. J. 76:199-202.

Hartl, D. L., and A. G. Clark. 1997. Principles of Population Genetics. 3rd ed. Sinauer Associates Inc., Sunderland, MA.

Hedrick, P. W. 2000. Genetics of Populations. Jones \& Bartlett Publishers, Sudbury, MA.

Hoeschele, I., and T. R. Meinert. 1990. Association of genetic defects with yield and type traits: The Weaver locus effect on yield. J. Dairy Sci. 73:2503-2515.

Hogasen, K., J. H. Jansen, and M. Harboe. 1997. Eradication of porcine factor $\mathrm{H}$ deficiency in Norway. Vet. Rec. 140:392-395.

Jansen, J., A. van Laarhoven, and E. W. Brascamp. 1984. Selection against undesirable recessive genes in A.I. cattle populations with overlapping generations. Z. Tierz. Zuechtungsbiol. 101:220-228.
Jolly, R. D. 1975. Mannosidosis of Angus cattle: A prototype control program for some genetic diseases. Adv. Vet. Sci. Comp. Med. 19:1-21.

Jolly, R. D., K. G. Thompson, and C. A. Tse. 1974. Evaluation of a screening programme for identification of mannosidosis heterozygotes in Angus cattle. N. Z. Vet. J. 22:185-190.

Jolly, R. D., and R. J. Townsley. 1980. Genetic screening programmes: an analysis of benefits and costs using the bovine mannosidosis scheme as a model. N. Z. Vet. J. 28:3-6.

Kerr, R. J., and B. P. Kinghorn. 1996. An efficient algorithm for segregation analysis in large populations. J. Anim. Breed. Genet. 113:457-469.

Kinghorn, B. P. 1999. Use of segregation analysis to reduce genotyping costs. J. Anim. Breed. Genet. 116:175-180.

Kraner, S., J. P. Sieb, P. N. Thompson, and O. K. Steinlein. 2002. Congenital myasthenia in Brahman calves caused by homozygosity for a CHRNE truncating mutation. Neurogenetics 4:87-91.

Leach, L. M., M. Ellis, D. S. Sutton, F. K. McKeith, and E. R. Wilson. 1996. The growth performance, carcass characteristics, and meat quality of halothane carrier and negative pigs. J. Anim. Sci. 74:934-943.

Lefort, G., and J. J. Lauvergne. 1974. L'eradication des genes recessifs a effets visibles dans des populations bovines ous les taureaux sont mis a l'epreuve sur leur descendance. C. R. Hebd. Seances Acad. Sci. Series D, Sci. Nat. 279:1381-1384.

Lincoln, S., J. Wiley, T. Lynch, J. W. Langston, R. Chen, A. Lang, E. Rogaeva, D. S. Sa, R. P. Munhoz, J. Harris, K. Marder, C. Klein, G. Bisceglio, J. Hussey, A. West, M. Hulihan, J. Hardy, and M. Farrer. 2003. Parkin-proven disease: Common founders but divergent phenotypes. Neurology 60:1605-1610.

Moelich, E. I., L. C. Hoffman, and P. J. Conradie. 2003. Sensory and functional meat quality characteristics of pork derived from three halothane genotypes. Meat Sci. 63:333-338.

Moran, P. A. P. 1958. A general theory of the distribution of gene frequencies. II. Non-overlapping generations. Proc. R. Soc. Lond. B. Biol. Sci. 149:113-116.

Nicholas, F. W. 2005. Online Mendelian Inheritance in Animals. Available: http://omia.angis.org.au Accessed Nov. 21, 2005.

Powell, R. L., H. D. Norman, and C. M. Cowan. 1996. Relationship of bovine leukocyte adhesion deficiency with genetic merit for performance traits. J. Dairy Sci. 79:895-899.

Robinson, J. L., R. G. Popp, R. D. Shanks, A. Oosterhof, and J. H. Veerkamp. 1993. Testing for deficiency of uridine monophosphate synthase among Holstein-Friesian cattle of North America and Europe. Livest. Prod. Sci. 36:287-298.

Schroeder, S. A., D. M. Gaughan, and M. Swift. 1995. Protection against bronchial asthma by CFTR delta F508 mutation: A heterozygote advantage in cystic fibrosis. Nat. Med. 1:703-705.

Shanks, R. D., and J. L. Robinson. 1989. Embryonic mortality attributed to inherited deficiency of uridine monophosphate synthase. J. Dairy Sci. 72:3035-3039.

Sonesson, A. K., L. L. Janss, and T. H. Meuwissen. 2003. Selection against genetic defects in conservation schemes while controlling inbreeding. Genet. Sel. Evol. 35:353-368. 